\title{
Identification of critical nodes in large-scale spatial networks *
}

\author{
Vishaal Krishnan Sonia Martínez ${ }^{\dagger}$
}

\begin{abstract}
The notion of network connectivity is used to characterize the robustness and failure tolerance of networks, with high connectivity being a desirable feature. In this paper, we develop a novel dynamical approach to the problem of identifying critical nodes in largescale networks, with algebraic connectivity (the second smallest eigenvalue of the graph Laplacian) as the chosen metric. Employing a graph-embedding technique, we reduce the class of considered weight-balanced graphs to spatial networks with uniformly distributed nodes and nearest-neighbors communication topologies. Through a continuum approximation, we consider the Laplace operator on a manifold (with the Neumann boundary condition) as the limiting case of the graph Laplacian. We then reduce the critical node set identification problem to that of finding a ball of fixed radius, whose removal minimizes the second (Neumann) eigenvalue of the Laplace operator on the residual domain. This leads us to consider two functional and nested optimization problems. Resorting to the Min-max theorem, we first treat the problem of determining the second smallest eigenvalue for a fixed domain by minimizing an energy functional. We then obtain a closed-form expression for a projected gradient flow that converges to the set of points satisfying the KKT conditions and provide a novel proof that the only locally asymptotically stable critical point is the second eigenfunction of the Laplace operator. Building on these results, we consider the critical ball identification problem and define novel dynamics to converge asymptotically to these points. Finally, we provide a characterization of the location of critical nodes (for infinitesimally-small balls) as those points which belong to the nodal set of the second eigenfunction of the Laplacian operator.
\end{abstract}

\section{Introduction}

The identification of critical nodes in a network is motivated by the question of network robustness and is crucial to improving its resilience to attacks and failures. The notion of critical nodes refers to the subset of nodes in the network whose removal results in the maximum deterioration of a given performance metric. In the context of robustness of networks/graphs, a widely studied metric [1,2] is the second smallest eigenvalue of the graph Laplacian matrix (also called the algebraic connectivity of the graph). In addition to being an indicator of how well connected the graph is, it is typically of significance in the context of agreement dynamics on networks (such as consensus and synchronization), as it governs the convergence rate of the dynamics.

\footnotetext{
${ }^{*}$ This work has been partially supported by grant FA9550-18-1-0158.

${ }^{\dagger}$ The authors are with the Department of Mechanical and Aerospace Engineering, University of California at San Diego, La Jolla CA 92093 USA (email: v6krishn@ucsd.edu; soniamd@ucsd.edu).
} 
The problem of identifying critical nodes in a network graph leads to combinatorial optimization problems. Thus, for large-scale networks any algorithm that solves the problem exactly is of high complexity. Motivated by this, we study a relaxation of the problem through a continuum approximation of the network to the spatial domain where the nodes are distributed.

Literature review. We first cite some works that present combinatorial approaches to the problem of critical node identification. In [3 6$]$, the authors investigate the problem of identifying nodes whose deletion minimizes some network connectivity metric. An alternative approach to improving network robustness involves incorporating redundancy in the network by adding nodes and links, also called network augmentation [7]. In [8, the authors study the problem of network design as a function of the comparative costs of augmentation and defense against attack/failure.

The approximation of large networks by weighted graphs over a continuum set of infinite cardinality appears in previous literature. In this way, in [9] large networks are approximated by the so-called graphons, which result from the limit of convergent sequences of large dense graphs. Extending this idea to spatial networks, where the nodes are embedded in a domain $\Omega \in \mathbb{R}^{N}$, the nodes can be thought to be indexed by their positions $x \in \Omega$, and interactions restricted between the nearest spatial neighbors. Combining these notions in the context of network consensus dynamics, the object of interest is the continuum counterpart of the graph Laplacian, the Laplace operator on the domain. Theoretical results concerning the convergence of the graph Laplacian to the Laplace operator can be found in [10] and [11], which motivates the approach adopted in this paper.

There have been severals attempts to investigate problems linking the shape of a domain with the sequence of eigenvalues of the Laplace operator, for various boundary conditions, although those related to the critical subset identification are fewer in number. The work 12 contains an overview of the literature on extremum problems for eigenvalues of elliptic (e.g. Laplace) operators. In 13, the authors consider the problem of placing small holes in a domain to optimize the smallest Neumann eigenvalue of the Laplace operator (but with Dirichlet boundary condition on the hole).

Statement of Contributions. In this paper, we aim to study a critical node set identification problem for large-scale spatial networks with an associated weight-balanced Laplacian matrix. By considering a graph embedding technique, we reduce the problem to spatial networks with uniformly distributed nodes and nearest-neighbors communication topologies. Then we consider a special case of a hole-placement problem, which consists of identifying the optimal location of the center of a ball in the domain that minimizes the smallest positive eigenvalue of the Laplace operator for the residual domain. With the help of the Min-max theorem, we formulate our objective as an infinite-dimensional, non-convex and nested optimization problem. This limits our goal at the outset to achieving convergence to a local optimum. Since the solution is hard to obtain analytically, we develop an algorithmic approach to such problem. First, we consider the inner optimization or eigenvalue problem, whose KKT points include the eigenvalues of the Laplace operator. We then provide a closed-form expression for the projected gradient flow in a Banach space for this problem that converges to the set of KKT points. Exploiting further the special properties of these dynamics, we prove that the only locally asymptotically stable equilibrium point for the dynamics is the second eigenfunction of the Laplace operator. Moreover, since the other KKT points are saddle 
points that are non-degenerate, we infer almost global asymptotic stability of the second eigenfunction. Building on these results, we then design a novel hole-placement dynamics for the nested-optimization problem, and prove its local asymptotic stability to strict local minima. Finally, we provide a characterization of critical balls in the interior of the domain, and study the limiting case when its radius approaches zero. We conclude that the location of such critical nodes is at the nodal set of the second eigenfunction of the Laplace operator, which has an intuitive geometric interpretation in some cases. A partial account of the results of this paper were presented without technical proofs in [14]. In addition to presenting the full technical proofs, we present further analysis on the limiting case of hole-placement problem and include additional simulation examples.

Organization. This paper is organized as follows. Sections 2 and 3 introduce some notation and preliminaries respectively. This is followed by the problem formulation in Section 4 and main analysis in Section 5. We present some simulation results in Section 6 and conclude with the summary and future directions in Section 7 .

\section{Notation}

We now introduce some basic notation used in the sequel. First, we denote by $\mathbf{1}_{n}$ the vector of ones $\mathbf{1}_{n}^{\top}=(1, \ldots, 1)^{\top} \in \mathbb{R}^{n}$, for some $n \in \mathbb{N}$. For a graph $G$, we denote by $L(G)$ the graph Laplacian and by $\lambda_{2}(L(G))$ the algebraic connectivity of the graph. The corresponding eigenvector, also called the Fiedler eigenvector, is denoted by $v^{F}$. The open ball of radius $r>0$ and centered at $x \in \mathbb{R}^{N}$ is represented by $B_{r}(x)$, and $|\Omega|$ denotes the Lebesgue measure of the set $\Omega \subset \mathbb{R}^{N}$. The set of square-integrable functions on $\Omega$ is denoted by $L^{2}(\Omega)$. In other words, $L^{2}(\Omega)=\left\{f:\left.\Omega \rightarrow \mathbb{R}\left|\int_{\Omega}\right| f\right|^{2} d \nu<\infty\right\}$, where $d v$ is the standard Lebesgue measure. When clear from the context, we will denote $\int_{\Omega} f d \nu$ simply as $\int_{\Omega} f$, for some $f \in L^{2}(\Omega)$, with a slight abuse of notation. For $f, g \in L^{2}(\Omega)$, we let $\langle f, g\rangle=\int_{\Omega} f g d \nu$ denote the inner product and $\|f\|^{2}=\langle f, f\rangle$ denote the corresponding induced norm. We denote by $H^{1}(\Omega)=\left\{\left.f \in L^{2}(\Omega)\left|\int_{\Omega}\right| \nabla f\right|^{2} d \nu<\infty\right\}$. For a bounded domain $\Omega$, we denote by $\partial \Omega$ the boundary of $\Omega$ and by $\mathbf{n}$ the outward normal to the boundary. We also let $S$ denote the Lebesgue measure on the boundary (where the integral of $f$ on the boundary is written as $\left.\int_{\partial \Omega} f d S\right)$. Let $\partial$ denote the partial differential operator. For a differentiable function $F: \Omega \times \Omega \rightarrow \mathbb{R}$, we denote by $\partial_{1} F\left(x_{0}, y_{0}\right)$ (resp. $\partial_{2} F\left(x_{0}, y_{0}\right)$ ) the partial derivative of $F$ w.r.t. the first argument (resp. the second argument), evaluated at $\left(x_{0}, y_{0}\right)$. Finally, given $\Omega \subset \mathbb{R}^{N}, \Delta(\Omega)$ represents the Laplace operator on the domain $\Omega$ (we omit $\Omega$ in $\Delta(\Omega)$ when it is clear from context).

\section{Preliminaries}

In this section, we present the necessary background for setting up the critical node identification problem addressed in this paper. We begin by explaining how we employ a graph embedding along with a continuum approximation to go from the graph Laplacian to the Laplace operator on the domain. Using the Min-max theorem, we are then able to characterize the second eigenvalue of the Laplace operator corresponding to the algebraic connectivity of the graph. We finally point out to a connection to agreement algorithms in networked systems. 
Let $G=(V, E)$ be a weight-balanced directed graph such that $|V|=n$, and $w_{i j}$ be the edge weight corresponding to $(i, j) \in E$. A map $\mathbf{x}: V \rightarrow \Omega \subset \mathbb{R}^{N}$, is called a graph embedding ( $N \ll n$ and $\Omega$ bounded), if $x_{i}=\mathbf{x}(i) \in \mathbb{R}^{N}$ is the (spatial) position assigned to node $i \in V$, and the map $\mathbf{x}$ preserves some proximity measure on the graph $G$. There exists a vast literature on graph embeddings 15,16 , of which we adopt the notion of the structurepreserving embedding. Starting with the unweighted, undirected graph corresponding to $G$ (where the weighted directed edges in $G$ are replaced by unweighted undirected edges), a structure preserving embedding can be constructed such that any node $j$ which is a neighbor of $i$ in the graph $G$ is within a ball of radius $h$ centered at at $x_{i}$ in the embedding. Once the graph is embedded in $\Omega \subset \mathbb{R}^{N}$, we view the nodes $V$ as having been sampled from an underlying distribution $\mu \in \mathcal{P}(\Omega)$ (with density function $\rho$, such that $d \mu=\rho$ dvol). It is always possible to obtain the weighted adjacency matrix $W=\left[w_{i j}\right]$ of the digraph $G$ as the discretization of a smooth weight function $\mathcal{W}: \Omega \times \Omega \rightarrow \mathbb{R}_{\geq 0}$, such that $w_{i j}=\mathcal{W}\left(x_{i}, x_{j}\right)$. The weight function $\mathcal{W}$ encodes the weights and directionality of the edges, and since the number of nodes $V$ is finite, such a smooth weight function always exists. Let $\varphi: \Omega \rightarrow \mathbb{R}$ be a real-valued function on $\Omega$ and $\phi^{d}: V \rightarrow \mathbb{R}$ such that $\phi_{i}^{d}=\phi^{d}(i)=\varphi\left(x_{i}\right)$. We define the $\mathcal{W}$-weighted average variation in $\varphi$ around a point $x \in \Omega$, averaged over a ball $B_{h}(x)$ of radius $h>0$ and centered at $x$ as follows:

$$
\frac{1}{\mu\left(B_{h}(x)\right)} \int_{B_{h}(x)} \mathcal{W}(x, y)(\varphi(y)-\varphi(x)) d \mu(y) .
$$

We see next that the weighted Laplace operator on $\Omega$ can be obtained as the limit of a $\mathcal{W}$ weighted average variation as $h \rightarrow 0$. We first let $w(x)=\mathcal{W}(x, x)$ and $\nabla w(x)=\frac{1}{2}\left(\partial_{1} \mathcal{W}+\right.$ $\left.\partial_{2} \mathcal{W}\right)(x, x)$, and we obtain the following by means of a Taylor expansion:

$$
\begin{array}{r}
\lim _{h \rightarrow 0} \frac{c}{h^{2}} \frac{1}{\mu\left(B_{h}(x)\right)} \int_{B_{h}(x)} \mathcal{W}(x, y)(\varphi(y)-\varphi(x)) d \mu(y) \\
=\frac{1}{\rho} \nabla \cdot(w \rho \nabla \varphi),
\end{array}
$$

where $c$ is a constant. The graph Laplacian matrix $L(G)$ corresponding to $G$ can now be viewed as the discretization of the (negative) $w$-weighted Laplace operator $-\frac{1}{\rho} \nabla \cdot(w \rho \nabla)$. Alternatively, the $w$-weighted Laplace operator can be viewed as an approximation of $L(G)$, with closer approximations obtained as $n=|V| \rightarrow \infty$ and $h \rightarrow 0$.

In addition, approximating the Laplacian matrix $L(G)$ by the Laplace operator on $\Omega$ requires the specification of a boundary condition. This condition is obtained by observing that $\mathbf{1}_{n} \in \operatorname{Null}\left(L^{\top}(G)\right)$, that is, $\left\langle\mathbf{1}, L(G) \phi^{d}\right\rangle=\mathbf{1}_{n}^{\top} L(G) \phi^{d}=0$ for any $\phi^{d}$. In the continuous setting, this translates into the Neumann boundary condition $\nabla \varphi \cdot \mathbf{n}=0$ on $\partial \Omega$. This can be seen from an application of the Divergence theorem, that is, $\left\langle 1, \frac{1}{\rho} \nabla \cdot(w \rho \nabla \varphi)\right\rangle=$ $\int_{\Omega} \frac{1}{\rho} \nabla \cdot(w \rho \nabla \varphi) d \mu=\int_{\partial \Omega} w \rho \nabla \varphi \cdot \mathbf{n} d S=0$ (if $\nabla \varphi \cdot \mathbf{n}=0$ ). Thus, the Neumann boundary condition is imposed as the natural boundary condition here.

Remark 1. (Problem reduction to uniformly spatially embedded graphs). Based on the previous considerations, and without loss of generality, in the following we focus on networks that are spatially embedded in an open bounded domain $\Omega$ according to a uniform distribution (the distribution $\mu$ is uniform above) and such that the underlying graph is undirected and unweighted. Note that the following derivations are analogous for the case of a 
non-uniform $\mu$ and weight-balanced directed graph: all results carry through by keeping the weights $w$ and $\rho$ in the weighted Laplace operator.

The Laplace operator $\Delta$ with the Neumann boundary condition, has an infinite sequence of eigenvalues $0=\mu_{1} \leq \mu_{2} \leq \ldots \leq \mu_{m} \leq \ldots$, whose corresponding eigenfunctions $\left\{\psi_{i}\right\}_{i=1}^{\infty}$ form an orthonormal basis for $L^{2}(\Omega), 17$. Using the Min-max theorem [17] for the operator $\Delta$, one can determine:

$$
\mu_{2}(\Omega)=\inf _{\psi \in\left\{\psi_{1}\right\}^{\perp}} \frac{\langle\psi, \Delta \psi\rangle_{L^{2}(\Omega)}}{\langle\psi, \psi\rangle_{L^{2}(\Omega)}},
$$

where $\left\{\psi_{1}\right\}^{\perp}=\left\{\psi \in H^{1}(\Omega) \mid \psi \neq 0, \int_{\Omega} \psi_{1} \psi d \nu=0\right\}$, and $\psi_{1}$ is constant, the eigenfunction corresponding to $\mu_{1}=0$. This implies $\left\{\psi_{1}\right\}^{\perp}=\left\{\psi \in H^{1}(\Omega) \mid \int_{\Omega} \psi d \nu=0\right\}$. Thus, using the Divergence theorem, applying the Neumann boundary condition, and normalizing the functions, we obtain an equivalent reformulation of (1) as:

$$
\mu_{2}(\Omega)=\inf _{\substack{\psi \in H^{1}(\Omega), \int_{\Omega} \psi d \nu=0 \\ \int_{\Omega}|\psi|^{2} d \nu=1}} \int_{\Omega}|\nabla \psi|^{2} d \nu .
$$

Remark 2. (Connection to agreement algorithms). The second eigenvalue is also of relevance to Laplacian-based agreement/consensus algorithms in networked systems, as it governs the convergence rate of these algorithms.

\section{Problem Formulation}

We define in this section the notion of criticality adopted in this manuscript. We define critical nodes as those nodes in the graph whose removal results in the maximum deterioration in algebraic connectivity for the residual network, making them the most crucial nodes to be protected.

More precisely, this amounts to identifying a set $K^{*} \subset \Omega$ of given measure $\left|K^{*}\right|=c>0$ such that $\mu_{2}\left(\Omega \backslash K^{*}\right)$ is an infimum. The problem of identifying the critical nodes, $K^{*}$, can be formulated as:

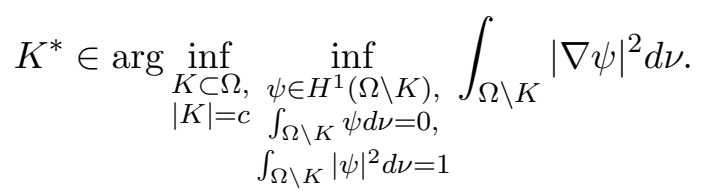

We restrict the search to a class of subsets $K=B_{r}(x)=\{y \in \Omega|| y-x \mid<r\} \subset \Omega$, open balls of radius $r$ (such that $\left|B_{r}(x)\right|=c$ ). This reduces the search space to $\tilde{\Omega}_{r}=\{x \in$ $\Omega \mid \operatorname{dist}(x, \partial \Omega)>r\}$, and the problem is reformulated as:

$$
x^{*} \in \arg \inf _{x \in \tilde{\Omega}_{r}} \inf _{\substack{\psi \in H^{1}\left(\Omega \backslash B_{r}(x)\right), \int_{\Omega \backslash B_{r}(x)} \psi d \nu=0, \int_{\Omega \backslash B_{r}(x)}|\psi|^{2} d \nu=1}}|\nabla \psi|_{\Omega}^{2} d \nu .
$$

which we refer to as the hole-placement problem in the sequel. 
Remark 3. (Generalization using multiple balls). We note that any compact subset $K \subset \Omega$ can be covered by a finite number $m$ of open balls of a given radius $r$, and with arbitrary precision (as $r \rightarrow 0$ and $m \rightarrow \infty$ ). Given a finite collection $\left\{B_{r}\left(x_{i}\right)\right\}_{i=1}^{m}$ of open balls, we can then formulate the above optimization w.r.t. $\left(x_{1}, \ldots, x_{m}\right)$, the positions of the $m$ open balls. For simplicity, we just focus on the one-ball case.

\section{$5 \quad$ Functional optimization to determine the most critical nodes}

Here, we present our main results and algorithms to determine the most critical nodes in the network, in a functional optimization framework. To do this, we begin with the eigenvalue problem (2) (which is the inner optimization problem in (3) ) for $D$, a fixed domain, and design a projected gradient flow to converge to a local minimizer of the problem. This algorithm will help us build subsequently the dynamics that can be employed to solve the full hole placement problem (3) in an algorithmic manner. The analysis of the projected gradient flow will also be instrumental in evaluating the properties of the second dynamics.

\subsection{Projected gradient flow to determine $\mu_{2}(\Omega)$}

In what follows, we study the eigenvalue problem (2), characterize its critical points, construct and analyze a novel projected gradient flow to converge to the infimum. We write the optimization problem (for the smallest positive eigenvalue of the Laplace operator on a domain $D$ with a $C^{1}$, Lipschitz boundary) as:

$$
\begin{aligned}
\inf _{\psi \in H^{1}(D)} & \int_{D}|\nabla \psi|^{2}, \\
\text { s.t } & \int_{D}|\psi|^{2}=1, \quad \int_{D} \psi=0, \\
& \nabla \psi \cdot \mathbf{n}=0 \text { on } \partial D .
\end{aligned}
$$

Let $\mathcal{S}_{D}=\left\{\left.\psi \in H^{1}(D)\left|\int_{D}\right| \psi\right|^{2}=1, \int_{D} \psi=0, \nabla \psi \cdot \mathbf{n}=0\right.$ on $\left.\partial D\right\}$ and $J(\psi)=\int_{D}|\nabla \psi|^{2}$. We can now express the above problem as $\inf _{\psi \in \mathcal{S}_{D}} J(\psi)$.

Lemma 1. (Minimizer of $J(\psi)$ ). The eigenfunctions of $\Delta(D)$ are the critical points of the functional $J(\psi)$, and the second eigenfunction $\psi_{2}$ of $\Delta(D)$ is the only minimizer of the functional $J(\psi)$ in $\mathcal{S}_{D}$. Moreover, the critical points of $J(\psi)$ are non-degenerate, i.e., the Hessian of $J(\psi)$ is non-singular at the critical points.

The content of this Lemma follows from the Min-max theorem [17]. We refer the reader to the Appendix for an alternative proof of this lemma, as well as for the proofs of other results contained in this paper. We explicitly compute the analytical expression for the Hessian of the objective function $J(\psi)$ in the proof of Lemma 1, which allows us to infer the non-degeneracy of the saddle points of $J(\psi)$ which is useful in establishing almost-global convergence of the projected gradient flow we present below.

We now provide a novel closed-form expression for a projected gradient flow to converge to the minimum value of $J(\psi)$ in $\mathcal{S}_{D}$. For smooth one-parameter families of functions 
$\{\psi(t, x)\}_{t \in \mathbb{R}_{\geq 0}}$ (with $x \in D$ ), the derivative of the objective functional $J$ is given by:

$$
\frac{d}{d t}[J(\psi(t))]=2 \int_{D} \nabla \psi \cdot \nabla\left(\partial_{t} \psi\right)=-2 \int_{D} \partial_{t} \psi(\Delta \psi)
$$

We obtain a gradient flow by setting $\partial_{t} \psi=\Delta \psi$. We project this flow onto the tangent space of the set $\mathcal{S}_{D}$. For $\psi \in \mathcal{S}_{D}$, we require that $\left\langle\psi, \partial_{t} \psi\right\rangle=0$ and $\int_{D} \partial_{t} \psi=0$, which are satisfied if (this will be shown in Proposition 1):

$$
\partial_{t} \psi=\Delta \psi-\frac{\langle\Delta \psi, \psi\rangle}{\|\psi\|^{2}} \psi=\Delta \psi-\langle\Delta \psi, \psi\rangle \psi
$$

since $\|\psi\|=1$ for $\psi \in \mathcal{S}_{D}$. Further, using $J(\psi)=-\langle\Delta \psi, \psi\rangle$, we get the projected gradient flow:

$$
\partial_{t} \psi=\Delta \psi+J(\psi) \psi
$$

The equilibria $\psi^{*}$ of (4) satisfy $\Delta \psi^{*}+J\left(\psi^{*}\right) \psi^{*}=0$ and the Neumann boundary condition $\nabla \psi^{*}=0$ on $\partial D$. Clearly, $J\left(\psi^{*}\right)$ is an eigenvalue, and so let $\mu^{*}=J\left(\psi^{*}\right)$. It is also clear that the equilibria of the projected gradient flow are also the critical points of the functional $J$ over the set $\mathcal{S}_{D}$.

Proposition 1. (Convergence of gradient flow). The set $\mathcal{S}_{D}$ is invariant with respect to the flow (4), and the solutions to (4) in $\mathcal{S}_{D}$ converge in an $L^{2}$ sense to the set of equilibria of (4). Moreover, the only locally asymptotically stable equilibrium in $\mathcal{S}_{D}$ for (4) is the second eigenfunction $\psi_{2}$.

Remark 4. (Implication of Proposition 1). Proposition 1 states that we have global convergence to the set of isolated equilibria of the gradient flow (4) and that only the second eigenfunction $\psi_{2}$ is locally asymptotically stable among the set of isolated equilibria. Moreover, as seen in the proof of Lemma 1, we have that the other equilibria are saddle points of $J(\psi)$ and are non-degenerate (the Hessian of $J$ at these saddle points are non-singular). From this we deduce almost global asymptotic stability of the second eigenfuction $\psi_{2}$ for the flow (4), and we therefore have convergence from almost all initial conditions, see [18] for an overview of this property.

\subsection{Design of hole-placement dynamics}

We now consider the full optimization problem (3), which can be expressed as:

$$
x^{*} \in \arg \inf _{x \in \tilde{\Omega}_{r}} \mu_{2}\left(\Omega \backslash B_{r}(x)\right)
$$

Assumption 1. (Simplicity of the second eigenvalue). We assume that the second eigenvalue $\mu_{2}\left(\Omega \backslash B_{r}(x)\right)$ is simple for any $x \in \tilde{\Omega}$.

Remark 5. (Relaxing Assumption 1). The assumption that the eigenvalue $\mu_{2}$ is simple is ensures differentiability of $\mu_{2}\left(\Omega \backslash B_{r}(x)\right)$ w.r.t. $x$. The eigenvalues of $\Delta\left(\Omega \backslash B_{r}(x)\right)$ exist as branches $x \mapsto \mu\left(\Omega \backslash B_{r}(x)\right)$, which can then be ordered as $\mu_{1} \leq \mu_{2} \leq \ldots$ for any given $x$. The branches $x \mapsto \mu\left(\Omega \backslash B_{r}(x)\right)$ of eigenvalues are differentiable w.r.t. $x$ (more generally 
w.r.t. the perturbation of domains with Lipschitz boundaries [12]). The case of a non-simple eigenvalue $\mu_{2}$ occurs when multiple branches intersect, for some $x$, at which point the ordering of the branches may change and we lose differentiability of $\mu_{2}$. This situation can however be mitigated by considering the subdifferential of $\mu_{2}$ in place of the gradient of $\mu_{2}$. The dynamics presented later in the paper can be modified in this sense, and the analysis would require further investigation on the regularity/lower-semicontinuity properties of these subdifferentials. We nevertheless avoid this problem through Assumption 1, which we leave as future work.

The following lemma allows for a characterization of the critical points of the functional $\mu_{2}$ in the interior of the domain.

Lemma 2. (Characterization of critical ball). The first-order condition for a critical point $x^{*}$ of the functional $\mu_{2}$ in the interior of the domain is given by:

$$
\mu_{2}^{*}\left(\int_{\partial B_{r}\left(x^{*}\right)}\left|\psi_{2}^{*}\right|^{2} \mathbf{n}\right)=\int_{\partial B_{r}\left(x^{*}\right)}\left|\nabla \psi_{2}^{*}\right|^{2} \mathbf{n},
$$

where $\left(\mu_{2}^{*}, \psi_{2}^{*}\right)$ is the second eigenpair such that $\mu_{2}^{*} \triangleq \mu_{2}\left(\Omega \backslash B_{r}\left(x^{*}\right)\right)$.

We now construct the gradient dynamics to converge to a critical point of $\mu_{2}$ in the interior of the domain. Note that the function $\mu_{2}\left(\Omega \backslash B_{r}(x)\right)$ is not known explicitly for a general domain $\Omega \backslash B_{r}(x)$. We reformulate the optimization problem (3) as:

$$
x^{*}=\arg _{1} \inf _{(x, \psi) \in \tilde{\Omega} \times \Psi(x)} \int_{\Omega \backslash B_{r}(x)}|\nabla \psi|^{2} d \nu
$$

where the set $\Psi(x)$ is defined as:

$$
\Psi(x)=\left\{\left.\psi \in H^{1}\left(\Omega \backslash B_{r}(x)\right)\left|\int_{\Omega \backslash B_{r}(x)} \psi=0, \int_{\Omega \backslash B_{r}(x)}\right| \psi\right|^{2}=1\right\},
$$

where $\arg _{1}$ indicates the first argument $x$ in $(x, \psi)$. We also define the set $\Psi=\cup_{x \in \tilde{\Omega}_{r}} \Psi(x)$. We recall that $\tilde{\Omega}_{r}=\{x \in \Omega \mid \operatorname{dist}(x, \partial \Omega)>r\}$. Now let $\{x(t)\}_{t \in \mathbb{R}_{\geq 0}}$ be a smooth curve in $\tilde{\Omega}_{r}$ and $\{\psi(t, y)\}_{t \in \mathbb{R}_{\geq 0}}$ (with $y \in \Omega \backslash B_{r}(x(t))$,) a smooth one-parameter family of functions on $\Omega \backslash B_{r}(x(t))$. Also, let $\tilde{\mathbf{n}}(x)$ be the normal to the boundary $\partial \tilde{\Omega}_{r}$ at $x \in \partial \tilde{\Omega}_{r}$. We now consider the following hole-placement dynamics for our nested optimization problem:

$$
\begin{aligned}
& \frac{d x}{d t}=\left\{\begin{array}{l}
\mathbf{v}_{i n t}, x \in \operatorname{int} \tilde{\Omega}_{r} \\
\mathbf{v}_{i n t}-\left(\mathbf{v}_{i n t} \cdot \tilde{\mathbf{n}}\right) \tilde{\mathbf{n}}, x \in \partial \tilde{\Omega}_{r}
\end{array}\right. \\
& \mathbf{v}_{i n t}=-\int_{\partial B_{r}(x)}|\nabla \psi|^{2} \mathbf{n}+J(\psi) \int_{\partial B_{r}(x)}|\psi|^{2} \mathbf{n}, \\
& \partial_{t} \psi=\Delta \psi+J(\psi) \psi+a \psi+b, \\
& \nabla \psi \cdot \mathbf{n}=0, \quad \text { on } \partial \Omega \cup \partial B_{r}(x),
\end{aligned}
$$

where $a=-\frac{1}{2} \mathbf{v} \cdot\left(\int_{\partial B_{r}(x)}|\psi|^{2} \mathbf{n}\right)$ and $b=-\frac{1}{|\Omega|-c} \mathbf{v} \cdot\left(\int_{\partial B_{r}(x)} \psi \mathbf{n}\right)$, with $c=\left|B_{r}(x)\right|$, for all $x \in \tilde{\Omega}_{r}$. 
Theorem 1. (Convergence of the hole placement dynamics). The set $\Psi$ in (7) is invariant with respect to the dynamics (8). The solutions to the dynamics (8) converge to a critical point of the objective functional $\mu_{2}$ in (6). A critical point of $\mu_{2}$ is locally asymptotically stable with respect to the dynamics (8) only if it is a strict local minimum.

Remark 6. (Implication of Theorem 1). Theorem 1 states that we have convergence to the equilibria of the hole-placement dynamics which are also critical points of $\mu_{2}\left(\Omega \backslash B_{r}(x)\right)$. In addition, we have that among the critical points of $\mu_{2}\left(\Omega \backslash B_{r}(x)\right)$, only the strict local minima are locally asymptotically stable. For almost global convergence to these strict local minima, we additionally require non-degeneracy of the saddle points of $\mu_{2}\left(\Omega \backslash B_{r}(x)\right.$ ) (i.e., that the Hessian is non-singular at the critical point), but this additional characterization is not contained in our result.

We now consider the following question: if an initial failure happens with the removal of a node, what is the most critical node? This is appropriately posed in the continuum setting as the hole placement problem where the size of the hole is very small, i.e., as the radius $r \rightarrow 0$. For this, we investigate the minimum of the function $f(x)=\lim _{r \rightarrow 0} \frac{1}{\partial B_{r}(x) \mid} \frac{\partial}{\partial r} \mu_{2}\left(\Omega \backslash B_{r}(x)\right)$, which quantifies as a function of the hole position, the rate of deterioration of the metric as failure begins to occur.

Theorem 2. (Connection to the nodal set of eigenfunction). In the limit $r \rightarrow 0$ for the radius of the hole, the hole-placement problem reduces to finding the minima $x^{*} \in \Omega$ of the function:

$$
f(x)=\mu_{2}^{\Omega}\left|\psi_{2}^{\Omega}(x)\right|^{2}-\left|\nabla \psi_{2}^{\Omega}(x)\right|^{2},
$$

where $\left(\mu_{2}^{\Omega}, \psi_{2}^{\Omega}(x)\right)$ is the second eigenpair of the domain $\Omega$. Moreover, if the family of level sets of $\psi_{2}^{\Omega}$ is locally flat at a point $x^{*} \in \Omega$, then $x^{*}$ is a local minimizer of $f$ if and only if $\psi_{2}^{\Omega}\left(x^{*}\right)=0$. In other words, under local flatness, the nodal points of $\psi_{2}^{\Omega}$ are the local minimizers of $f$.

Remark 7. (Geometry of nodal sets). The nodal sets of Neumann eigenfunctions have been extensively investigated [19]. It is known that if the domain is symmetric about a subset, then it contains the nodal set of $\psi_{2}$. The nodal set for the second eigenfunction $\psi_{2}^{\Omega}$ divides the domain $\Omega$ into no more than two regions $\Omega_{a}$ and $\Omega_{b}$. Now, $\mu_{2}^{\Omega}$ is the first eigenvalue $\lambda_{1}$ of the Laplacian for $\Omega_{a}$ and $\Omega_{b}$, with Neumann boundary condition on $\partial \Omega \cap \partial \Omega_{a}$ and Dirichlet boundary condition on $\partial \Omega_{a} \cap \partial \Omega_{b}$.

Remark 8. (Implication for networks). Theorem 2 can be used to provide new insight on where the most critical nodes in a network with a finite number of nodes are located, via a continuum approximation. This is based on the fact that the entries $v_{i}^{F}$ of the Fiedler eigenvector $v^{F}$ of the finite graph embedded in $\Omega$ can be approximated by the value of the eigenfunction $\psi_{2}^{\Omega}$ at the location $x_{i}$ of the node $i$. That is, $v_{i}^{F} \approx \psi_{2}^{\Omega}\left(x_{i}\right)$. Then the most critical nodes in the network are expected at the zero entries of the Fiedler eigenvector. The Fiedler eigenvector, however, does not necessarily contain zero entries for general finite graphs (this situation improves with the size of the graph), in which case we may expect the critical nodes to be concentrated at the entries of lowest magnitude. This is a heuristic obtained from the fact that $\psi_{2}^{\Omega}$ is smooth and that $\psi_{2}^{\Omega}$ more closely approximates $v^{F}$ as $n \rightarrow \infty$. 


\section{Simulation results}

In this section, we present some numerical simulation results that can illustrate the concepts and algorithms of the previous sections.

First, we consider a disk-shaped domain $\Omega$ of unit radius, and the placement of a hole $B$ of radius of 0.1 units. Figure 1 shows a plot of $\mu_{2}$ for the residual domain $\Omega \backslash B$ as a function of $h$ (distance between the center of the disk and the center of the hole). Since the hole is of radius 0.1 units and is contained in $\Omega$, we note that $h \in[0,0.9)$. We observe

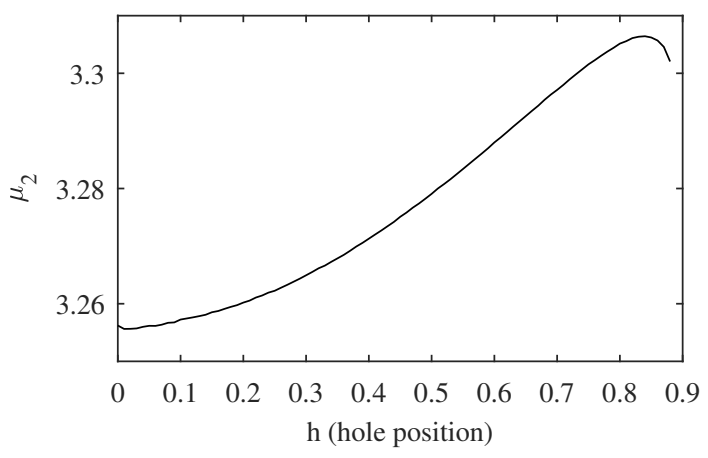

Figure 1: $\mu_{2}$ as a function of $h$ for a disk-shaped domain.

from Figure 1 that the second (also the smallest positive) eigenvalue of the Laplace operator for a disk-shaped domain with a hole increases with the distance between the centers of the domain and the hole, but also appears to decrease as the hole approaches close to the domain boundary (around $h=0.85$ units). Moreover, $\mu_{2}$ as a function of $h$ appears to be a convex in the interval $h \in[0,0.85]$ and concave for $h \in(0.85,0.9)$.

We now present simulation results for the projected gradient flow (8). For the simulation, we have separated the dynamics into two time scales, with $x$ (the center of the hole) as the slow-scale variable and $\psi$ the fast-scale variable. We first consider the case of the disk-shaped domain, that is, the dynamics (8) corresponds to hole placement for the disk-shaped domain to minimize $\mu_{2}$ of the residual domain.

Figure 2 is a plot of $x(t)$, the path of the center of the hole, on the spatial domain, for two different initial conditions $x(0)=(0.4,0.5)$ and $x(0)=(-0.5,-0.5)$. We observe that the hole center approaches the center of the disk with time, approximately along a straight line.

Figure 3 is a plot of $x(t)$, the path of the center of the hole (from the dynamics (8p) for a convex polygonal spatial domain. The final location of the hole is also indicated in the figure.

Figure 4 contains the results for a non-convex polygonal domain. The outer polygon is the spatial domain $\Omega$, while the inner polygon is the domain $\tilde{\Omega}$ (the set of allowed positions for the center of the hole). The heatmap shows the value of $\mu_{2}$ of the residual domain (which was obtained by first sampling the domain uniformly at random at the points indicated by the tiny circles, placing the hole at those points, computing $\mu_{2}$ of the residual domain, and then interpolating to obtain the plot). The paths of the center of the hole $x(t)$ (from the 


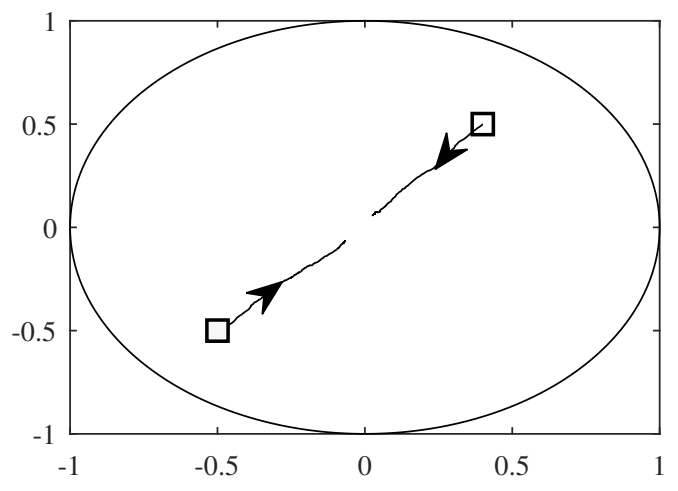

Figure 2: Path of the center of the hole, $x(t)$ from two different initial conditions $x(0)=$ $(0.4,0.5)$ and $x(0)=(-0.5,-0.5)$.

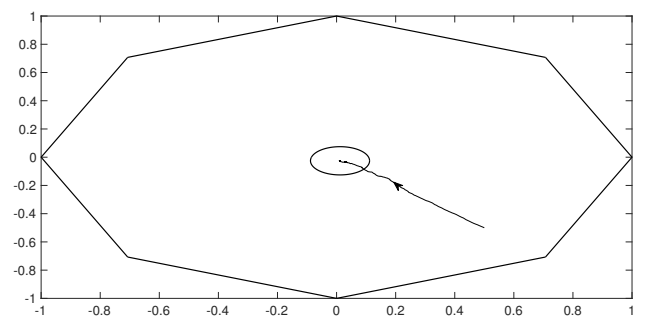

Figure 3: Path of the center of the hole, $x(t)$ from an initial condition $x(0)=(0.5,-0.5)$ for a convex polygonal domain.

dynamics (8)) from different initial conditions are also plotted. The paths do not all converge to the same point in this case, but to a broader region (the darker region in the heatmap), which possibly contains more than one local minimum $x^{*}$.

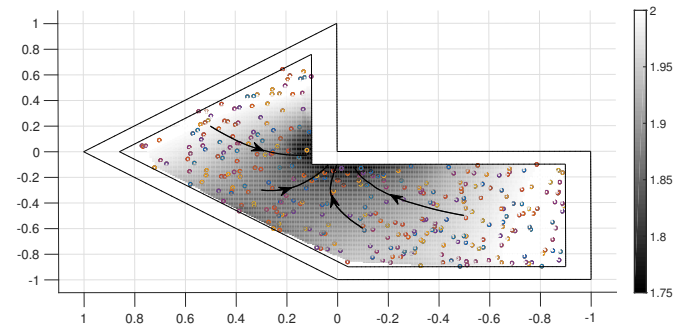

Figure 4: Paths of the center of the hole, $x(t)$ from different initial conditions.

In Figure 5, we present a numerical validation of the discussion in Remark 8, We first generated a random connected graph $G$ with 50 nodes. We then computed the algebraic connectivities of the residual graphs obtained by the removal of one node from the 
graph $\lambda_{2}(L(G \backslash\{i\}))$, for each node, plotting it against the corresponding entry of the Fiedler eigenvector $v_{i}^{F}$ (the eigenvector corresponding to the second eigenvalue of the Laplacian, or algebraic connectivity) of the original graph $G$. From the discussion in Remark 8, we expect that the local minima of $\lambda_{2}(L(G \backslash\{i\}))$ are concentrated around nodes corresponding to the entries of the Fiedler eigenvector of lowest magnitude, which is illustrated in the figure. We note that in the corresponding hole-placement problem, the nodal sets of the second eigenfunction $\psi_{2}^{\Omega}$ are only the local minimizers of $f(x)=\mu_{2}^{\Omega}\left|\psi_{2}^{\Omega}(x)\right|^{2}-\left|\nabla \psi_{2}^{\Omega}(x)\right|^{2}$. We thereby do not expect all the zero entries of the Fiedler eigenvector to correspond necessarily to global minimizers. However, the figure shows that the global minimum is indeed concentrated around nodes corresponding to the entries of the Fiedler eigenvector of lowest magnitude.

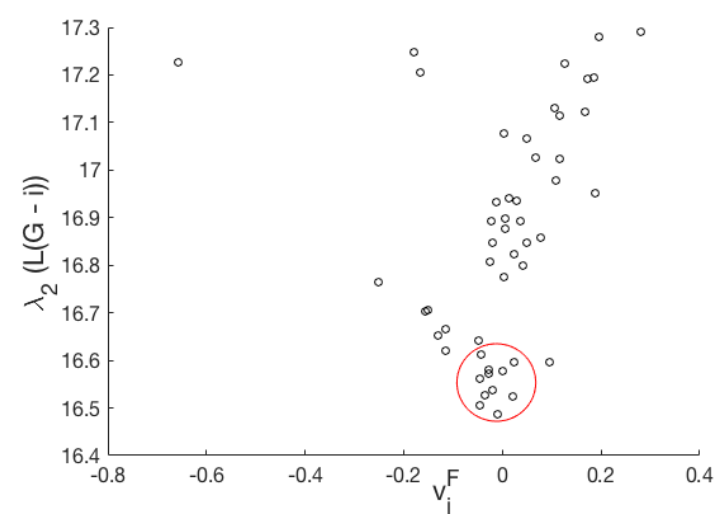

Figure 5: Plot of algebraic connectivity of residual network with the removal of one node vs. its corresponding entry in the Fiedler eigenvector, for a network with 50 nodes.

\section{Conclusions}

In this paper, we studied the problem of identifying the critical nodes for consensus in largescale spatial networks. We began by making a functional approximation of the Laplacian matrix of the graph to the Laplace operator on the domain. In addition to being a natural step in the large- $N$ limit, the real advantage of the approximation is that it does not conceal the geometry of the problem, which is important for spatial networks such as swarms and sensor networks. As a starting point, we analyzed the removal of balls of given measure from the domain. In future work, we would like to generalize the results to arbitrary sets over domains with a non uniform distribution of nodes. Further generalization of the analysis relaxing Assumption 1, as outlined in Remark 5, is also left for future work. We note that the proposed gradient dynamics were centralized in nature, the problem of distributed critical node set identification is also of interest and left for future work. 


\section{Appendix}

Proof. (Proof of Lemma 1). The first variation of the Lagrangian $L(\psi, \mu, \lambda)=J(\psi)+$ $\mu\left(1-\int_{D}|\psi|^{2}\right)+\lambda \int_{D} \psi$, at a critical point $\psi^{*}$ is zero (where $\int_{D}|\psi|^{2}=1$ and $\int_{D} \psi=0$ are the constraints, as $\psi \in \mathcal{S}_{D}$ and the Neumann boundary condition is assumed implicitly.) Thus, for any $\delta \psi \in T_{\psi^{*}} \mathcal{S}_{D}$ the tangent space of $\mathcal{S}_{D}$ at $\psi^{*}$, we have $\left\langle\frac{\delta L}{\delta \psi}, \delta \psi\right\rangle\left(\psi^{*}, \mu^{*}, \lambda^{*}\right)=$ $2 \int_{D} \nabla \psi^{*} \cdot \nabla(\delta \psi)-2 \mu^{*} \int_{D} \psi^{*} \delta \psi+\lambda^{*} \int_{D} \delta \psi=-2 \int_{D}\left(\Delta \psi^{*}+\mu^{*} \psi^{*}-\frac{1}{2} \lambda^{*}\right) \delta \psi=0$, for any $\delta \psi$ (note that the Neumann boundary condition was used in obtaining the equation.) Additionally, we also have $\left\langle\frac{\partial L}{\partial \mu}, \delta \mu\right\rangle\left(\psi^{*}, \mu^{*}, \lambda^{*}\right)=1-\int_{D}\left|\psi^{*}\right|^{2}=0$, and $\left\langle\frac{\partial L}{\partial \lambda}, \delta \lambda\right\rangle\left(\psi^{*}, \mu^{*}, \lambda^{*}\right)=\int_{D} \psi^{*}=0$. Thus, the critical points of the objective functional $\psi^{*} \in \mathcal{S}_{D}$ are characterized by:

$$
\Delta \psi^{*}+\mu^{*} \psi^{*}-\frac{1}{2} \lambda^{*}=0 .
$$

Integrating the previous equation over $D$ and using the Neumann boundary condition, we obtain $\lambda^{*}=0$. Therefore, the critical points $\psi^{*}$ satisfy:

$$
\Delta \psi^{*}+\mu^{*} \psi^{*}=0
$$

Let $\psi(x, \epsilon, \eta), x \in D$, be a smooth two-parameter family of functions in $\mathcal{S}_{D}$ with $\int_{D} \psi(x, \epsilon, \eta)=$ 0 for all $\epsilon$ and $\eta$. The first variation of $J$ at $\epsilon=0, \eta=0$ is given by:

$$
\left.\frac{\delta J}{\delta \epsilon}\right|_{\substack{\epsilon=0, \eta=0}}(\psi)=2 \int_{D} \nabla \psi \cdot \partial_{\epsilon} \nabla \psi=2 \int_{D} \nabla \psi \cdot \nabla\left(\partial_{\epsilon} \psi\right) .
$$

We let $\left.\partial_{\epsilon} \psi\right|_{\epsilon=0, \eta=0}=X$ and $\left.\partial_{\eta} \psi\right|_{\epsilon=0, \eta=0}=Y$. The second variation of $J$ at $\epsilon=0, \eta=0$ is given by:

$$
\begin{aligned}
\frac{\delta^{2} J}{\delta \eta \delta \epsilon}(X, Y) & =2 \int_{D} \nabla\left(\partial_{\eta} \psi\right) \cdot \nabla\left(\partial_{\epsilon} \psi\right)+2 \int_{D} \nabla \psi \cdot \nabla\left(\partial_{\eta \epsilon} \psi\right) \\
& =2 \int_{D} \nabla\left(\partial_{\eta} \psi\right) \cdot \nabla\left(\partial_{\epsilon} \psi\right)-2 \int_{D} \Delta \psi\left(\partial_{\eta \epsilon} \psi\right) \\
& =2 \int_{D} \nabla X \cdot \nabla Y-2 \int_{D} \Delta \psi\left(\partial_{\eta \epsilon} \psi\right) .
\end{aligned}
$$

Evaluating the second variation at a critical point $\psi(x, 0,0)=\psi^{*}$, and from $(9)$, we obtain:

$$
\frac{\delta^{2} J}{\delta \eta \delta \epsilon}(X, Y)=2 \int_{D} \nabla X \cdot \nabla Y+2 \mu^{*} \int_{D} \psi^{*}\left(\partial_{\eta \epsilon} \psi^{*}\right)
$$

Since $\psi(x, \epsilon, \eta)$ is a smooth two-parameter family of functions in $\mathcal{S}_{D}$, we have $\int_{D}|\psi(x, \epsilon, \eta)|^{2}=$ 1 for all $\epsilon, \eta$, which implies that $\int_{D} \psi\left(\partial_{\epsilon} \psi\right)=0$ and $\int_{D} \partial_{\eta} \psi \partial_{\epsilon} \psi+\int_{D} \psi\left(\partial_{\eta \epsilon} \psi\right)=\int_{D} X Y+$ $\int_{D} \psi\left(\partial_{\eta \epsilon} \psi\right)=0$. Substituting in 10$)$, we obtain:

$$
\frac{\delta^{2} J}{\delta \eta \delta \epsilon}(X, Y)=2 \int_{D} \nabla X \cdot \nabla Y-2 \mu^{*} \int_{D} X Y .
$$


In particular, for $X \neq 0$, this implies:

$$
\begin{aligned}
\frac{\delta^{2} J}{\delta \eta \delta \epsilon}(X, X) & =2 \int_{D}|\nabla X|^{2}-2 \mu^{*} \int_{D}|X|^{2} \\
& =2\left(\int_{D}|X|^{2}\right)\left(\frac{\int_{D}|\nabla X|^{2}}{\int_{D}|X|^{2}}-\mu^{*}\right) .
\end{aligned}
$$

We also have that $\int_{D} \psi(x, \epsilon, \eta)=0$, which leads to $\int_{D} \partial_{\epsilon} \psi=\int_{D} X=0$. From (2), we have that $\inf _{\int_{D} X=0} \frac{\int_{D}|\nabla X|^{2}}{\int_{D}|X|^{2}}=\mu_{2}$, which implies that if $\mu^{*}>\mu_{2}$ in (11), by the definition of infimum, there exists an $X$ such that $\left.\frac{\delta^{2} J}{\delta \eta \delta \epsilon}\right|_{\epsilon=0, \eta=0}(X, X)<0$. Therefore, the only critical point for which $\left.\frac{\delta^{2} J}{\delta \eta \delta \epsilon}\right|_{\epsilon=0, \eta=0}(X, X) \geq 0$ is the second eigenfunction $\psi^{*}=\psi_{2}$. Note that, for this case, $\left.\frac{\delta^{2} J}{\delta \eta \delta \epsilon}\right|_{\epsilon=0, \eta=0}(X, X)=0$ if and only if $X=k \psi_{2}$. Since $\int_{D} \psi_{2} X=0$, it must be that $k=0$, and therefore $X=0$. Thus, for all $X \neq 0,\left.\frac{\delta^{2} J}{\delta \eta \delta \epsilon}\right|_{\epsilon=0, \eta=0}(X, X)>0$ at $\psi^{*}=\psi_{2}$. Therefore, the second eigenfunction $\psi_{2}$ is the only minimizer of the functional $J(\psi)$ in $\mathcal{S}_{D}$. It further follows from the above argument that the Hessian $\left.\frac{\delta^{2} J}{\delta \eta \delta \epsilon}\right|_{\epsilon=0, \eta=0}$ is non-degenerate (or non-singular) at the critical points of $J(\psi)$, that is, $\left.\frac{\delta^{2} J}{\delta \eta \delta \epsilon}\right|_{\epsilon=0, \eta=0}(X, X)=0$ at the critical points of $J(\psi)$ if and only if $X=0$.

Proof. (Proof of Proposition 1). Recall that $\mathcal{S}_{D}=\left\{\left.\psi \in H^{1}(D)\left|\int_{D}\right| \psi\right|^{2}=1, \int_{D} \psi=0\right\}$. Therefore, for a smooth one-parameter family $\{\psi(t, x)\}_{t \in \mathbb{R}_{>0}}$, (with $x \in D$ ) to be in $\mathcal{S}_{D}$, we need to prove that $\int_{D} \psi \partial_{t} \psi=0$ and $\int_{D} \partial_{t} \psi=0$, assuming that the initial condition is in $\mathcal{S}_{D}$. (Note that it will later be shown that $\frac{d}{d t}\|\nabla \psi\| \leq 0$, thus $\psi(t, \cdot) \in H^{1}(D)$ for all $t \geq 0$ if $\left.\psi(0, \cdot) \in \mathcal{S}_{D}\right)$.

From Equation (4), we have $\int_{D} \psi \partial_{t} \psi=\int_{D} \psi(\Delta \psi+J(\psi) \psi)$. Using the Divergence theorem and the Neumann boundary condition on $\partial \Omega$, we get $\int_{D} \psi \partial_{t} \psi=-\int_{D}|\nabla \psi|^{2}+J(\psi) \int_{D}|\psi|^{2}=$ 0 (since $J(\psi)=\int_{D}|\nabla \psi|^{2}$ and $\int_{D}|\psi|^{2}=1$ ).

We also have $\int_{D} \partial_{t} \psi=\int_{D} \Delta \psi+J(\psi) \int_{D} \psi=\int_{D} \nabla \psi \cdot \mathbf{n}+J(\psi) \int_{D} \psi=0$ because of the Neumann boundary condition, $\nabla \psi \cdot \mathbf{n}=0$ on $\partial D$, and $\int_{D} \psi=0$.

Let $\psi(t, x)$ be a solution of (4) in $\mathcal{S}_{D}$, with $t \in \mathbb{R}_{\geq 0}, x \in D$, such that $\psi(0, x) \in \mathcal{S}_{D}$. We also have $\int_{D}|\psi|^{2}=1$, for all $t \geq 0$. Thus, $J(\psi)=\int_{D}|\nabla \psi|^{2}=\frac{\int_{D}|\nabla \psi|^{2}}{\int_{D}|\psi|^{2}}$. The time derivative 
of $J$ is given by:

$$
\begin{aligned}
\frac{d}{d t} J & =\frac{2}{\int_{D}|\psi|^{2}} \int_{D} \nabla \psi \cdot \nabla \partial_{t} \psi-2 \frac{\int_{D}|\nabla \psi|^{2}}{\left(\int_{D}|\psi|^{2}\right)^{2}} \int_{D} \psi \partial_{t} \psi \\
& =-2 \int_{D} \Delta \psi \partial_{t} \psi-2 J(\psi) \int_{D} \psi \partial_{t} \psi \\
& =-2 \int_{D}(\Delta \psi+J(\psi) \psi) \partial_{t} \psi \\
& =-2 \int_{D}|\Delta \psi+J(\psi) \psi|^{2} \leq 0 .
\end{aligned}
$$

We have that $J \geq 0$ and $\frac{d}{d t} J \leq 0$. We also have $\mathcal{S}_{D} \subset H^{1}(D), D$ a bounded, open subset of $\mathbb{R}^{N}$ with $\partial D$ being $C^{1}$. Thus by the Rellich-Kondrachov Compactness Theorem [17, we get that the orbit $\psi$ is precompact in $L^{2}(D)$. Therefore, by the LaSalle invariance principle for infinite dimensional spaces 20], the solutions converge in an $L^{2}$ sense to largest invariant set contained in $\left\{\psi^{*} \in \mathcal{S}_{D} \mid \Delta \psi^{*}+J\left(\psi^{*}\right) \psi^{*}=0\right\}$, the set of equilibria of (4).

In what follows we use the shorthand $\partial_{t} \psi=F(\psi)$, where $F\left(\psi^{*}\right)=0$, for the dynamics (4). We consider perturbations $\delta \psi \in \mathcal{T}_{D}$ along the tangent space of $\mathcal{S}_{D}$ at $\psi^{*}$ (also note that $\psi^{*}$ is an eigenfunction). Thus $\int_{D} \delta \psi=0$ and $\int_{D} \psi^{*} \delta \psi=0$. We have:

$$
F\left(\psi^{*}+\delta \psi\right)=\Delta\left(\psi^{*}+\delta \psi\right)+J\left(\psi^{*}+\delta \psi\right)\left(\psi^{*}+\delta \psi\right)
$$

Since $\psi^{*}$ is a critical point of $J(\psi)$ it holds that $J\left(\psi^{*}+\delta \psi\right)=J\left(\psi^{*}\right)+\mathcal{O}\left(\|\delta \psi\|^{2}\right)=\mu^{*}+$ $\mathcal{O}\left(\|\delta \psi\|^{2}\right)$. Thus, up to first-order we have that:

$$
\begin{aligned}
F\left(\psi^{*}+\delta \psi\right) & =\Delta\left(\psi^{*}+\delta \psi\right)+J\left(\psi^{*}+\delta \psi\right)\left(\psi^{*}+\delta \psi\right) \\
& =-\mu^{*} \psi^{*}+\Delta(\delta \psi)+\mu^{*} \psi^{*}+\mu^{*} \delta \psi \\
& =\Delta(\delta \psi)+\mu^{*} \delta \psi .
\end{aligned}
$$

Therefore, we have $\partial_{t}(\delta \psi)=\Delta(\delta \psi)+\mu^{*} \delta \psi$. Expressing $\delta \psi(t)=\sum_{i=2}^{\infty} \alpha_{i}(t) \psi_{i}$, where $\psi_{i}$ are the eigenfunctions which form an orthonormal basis for $\mathcal{T}_{D}$, we have that:

$$
\begin{aligned}
\partial_{t}(\delta \psi)=\sum_{i=2}^{\infty} \frac{d}{d t} \alpha_{i}(t) \psi_{i} & =\Delta(\delta \psi)+\mu^{*} \delta \psi \\
& =\sum_{i=2}^{\infty} \alpha_{i}(t)\left(-\mu_{i}+\mu^{*}\right) \psi_{i},
\end{aligned}
$$

which implies that $\delta \psi(t)=\sum_{i=2}^{\infty} e^{\left(\mu^{*}-\mu_{i}\right) t} \alpha_{i}(0) \psi_{i}$. (Note that, from orthogonality, the previous equality leads to $\frac{d}{d t} \alpha_{i}(t)=\alpha_{i}(t)\left(-\mu_{i}+\mu^{*}\right)$, for each $i$.) We claim that the latter converges to $\delta \psi=0$ for all initial conditions $\delta \psi(0) \in \mathcal{T}_{D}$ at $\psi^{*}$ if and only if $\mu^{*}=\mu_{2}$ (correspondingly, $\psi^{*}=\psi_{2}$ ). To see this, first observe that, if $\mu^{*}=\mu_{2}$ (correspondingly, $\psi^{*}=\psi_{2}$ ), we have $\int_{D} \psi_{2} \delta \psi(0)=0$ (since $\delta \psi \in \mathcal{T}_{D}$ at $\left.\psi^{*}=\psi_{2}\right)$, which implies that $\alpha_{2}(0)=\alpha_{2}(t)=0$. Hence $\delta \psi(t)=\sum_{i=3}^{\infty} e^{\mu_{2}-\mu_{i}} \alpha_{i}(0) \psi_{i}$ and the exponent $\mu_{2}-\mu_{i}<0$ for all $i \geq 3$. Conversely, if $\delta \psi(t)=\sum_{i=2}^{\infty} e^{\left(\mu^{*}-\mu_{i}\right) t} \alpha_{i}(0) \psi_{i}$ converges to $\delta \psi=0$ for all initial conditions $\delta \psi(0) \in \mathcal{T}_{D}$ at $\psi^{*}$, and $\psi^{*}=\psi_{i}$ for some $i \in\{2,3, \ldots\}$. We have that $\alpha_{i}(0)=\alpha_{i}(t)=0$ (from orthogonality), and that $\delta \psi(t)=\sum_{j=2, j \neq i}^{\infty} e^{\left(\mu_{i}-\mu_{j}\right) t} \alpha_{j}(0) \psi_{j}$, which converges to $\delta \psi=0$ only if $i=2$. Therefore, the second eigenfunction $\psi_{2}$ is the only locally asymptotically stable equilibrium in $\mathcal{S}_{D}$ for the projected gradient flow. 
Proof. (Proof of Lemma 2). Let $x(\epsilon)$ for $\epsilon \in \mathbb{R}$ be a smooth curve contained in $\tilde{\Omega}_{r}$. Let $\psi_{2}^{\epsilon}$ be the second eigenfunction of the Laplace operator with Neumann boundary condition in the domain $\Omega \backslash B_{r}(x(\epsilon))$. Thus, we have $\mu_{2}^{\epsilon}=\int_{\Omega_{\epsilon}}\left|\nabla \psi_{2}^{\epsilon}\right|^{2}$, where $\Omega_{\epsilon}=\Omega \backslash B_{r}(x(\epsilon))$ and $\left\|\psi_{2}^{\epsilon}\right\|_{\Omega_{\epsilon}=1}$. The derivative $\frac{d \mu_{2}^{\epsilon}}{d \epsilon}$ is given by:

$$
\frac{d \mu_{2}^{\epsilon}}{d \epsilon}=\frac{d}{d \epsilon} \int_{\Omega_{\epsilon}}\left|\nabla \psi_{2}^{\epsilon}\right|^{2}=2 \int_{\Omega_{\epsilon}} \nabla \psi_{2}^{\epsilon} \cdot \nabla\left(\frac{\partial \psi_{2}^{\epsilon}}{\partial \epsilon}\right)+\int_{\partial \Omega_{\epsilon}}\left|\nabla \psi_{2}^{\epsilon}\right|^{2} \mathbf{v} \cdot \mathbf{n}
$$

where $\mathbf{v}=\frac{d x(\epsilon)}{d \epsilon}$, is constant on $\partial B_{r}\left(x_{\epsilon}\right)$. Equation 12 becomes:

$$
\begin{aligned}
\frac{d \mu_{2}^{\epsilon}}{d \epsilon}= & 2 \int_{\Omega_{\epsilon}} \nabla \psi_{2}^{\epsilon} \cdot \nabla\left(\frac{\partial \psi_{2}^{\epsilon}}{\partial \epsilon}\right)+\mathbf{v} \cdot\left(\int_{\partial B_{r}\left(x_{\epsilon}\right)}\left|\nabla \psi_{2}^{\epsilon}\right|^{2} \mathbf{n}\right) \\
= & -2 \int_{\Omega_{\epsilon}} \frac{\partial \psi_{2}^{\epsilon}}{\partial \epsilon} \Delta \psi_{2}^{\epsilon}+\mathbf{v} \cdot\left(\int_{\partial B_{r}\left(x_{\epsilon}\right)}\left|\nabla \psi_{2}^{\epsilon}\right|^{2} \mathbf{n}\right) \\
= & 2 \int_{\Omega_{\epsilon}} \mu_{2}^{\epsilon} \psi_{2}^{\epsilon} \frac{\partial \psi_{2}^{\epsilon}}{\partial \epsilon}+\mathbf{v} \cdot\left(\int_{\partial B_{r}\left(x_{\epsilon}\right)}\left|\nabla \psi_{2}^{\epsilon}\right|^{2} \mathbf{n}\right) \\
= & \mu_{2}^{\epsilon} \frac{d}{d \epsilon}\left(\int_{\Omega_{\epsilon}}\left|\psi_{2}^{\epsilon}\right|^{2}\right)-\mu_{2}^{\epsilon} \mathbf{v} \cdot\left(\int_{\partial B_{r}\left(x_{\epsilon}\right)}\left|\psi_{2}^{\epsilon}\right|^{2} \mathbf{n}\right) \\
& +\mathbf{v} \cdot\left(\int_{\partial B_{r}\left(x_{\epsilon}\right)}\left|\nabla \psi_{2}^{\epsilon}\right|^{2} \mathbf{n}\right) \\
= & -\mu_{2}^{\epsilon} \mathbf{v} \cdot\left(\int_{\partial B_{r}\left(x_{\epsilon}\right)}\left|\psi_{2}^{\epsilon}\right|^{2} \mathbf{n}\right)+\mathbf{v} \cdot\left(\int_{\partial B_{r}\left(x_{\epsilon}\right)}\left|\nabla \psi_{2}^{\epsilon}\right|^{2} \mathbf{n}\right),
\end{aligned}
$$

since $\int_{\Omega_{\epsilon}}\left|\psi_{2}^{\epsilon}\right|^{2}=1$ for all $\epsilon \in \mathbb{R}$, which implies that $\frac{d}{d \epsilon}\left(\int_{\Omega_{\epsilon}}\left|\psi_{2}^{\epsilon}\right|^{2}\right)=0$. Let $x(0)=x^{*} \in \tilde{\Omega}$ be a critical point of $\mu_{2}(x)$, such that $\mu_{2}\left(x^{*}\right)=\mu_{2}^{*}$, with $\psi_{2}^{*}$ being the second eigenfunction. Thus we have $\left.\frac{d \mu_{2}^{\epsilon}}{d \epsilon}\right|_{\epsilon=0}=0$ for all $\mathbf{v}$, which implies that:

$$
\mu_{2}^{*}\left(\int_{\partial B_{r}\left(x^{*}\right)}\left|\psi_{2}^{*}\right|^{2} \mathbf{n}\right)=\int_{\partial B_{r}\left(x^{*}\right)}\left|\nabla \psi_{2}^{*}\right|^{2} \mathbf{n} .
$$

This is the first-order condition for critical points of $\mu_{2}$ in the interior of the domain.

Proof. (Proof of Theorem 1). Let $\{x(t), \psi(t, y)\}_{t \in \mathbb{R}_{>0}}$ (with $y \in \Omega \backslash B_{r}(x(t)$ ), ) be a one-parameter family of functions that is a solution to the dynamics (8), and let $\psi(0, \cdot) \in$ $\Psi(x(0))$. To prove the invariance of $\Psi(x(t))$, we need to show that $\frac{d}{d t}\left(\int_{\Omega \backslash B_{r}(x(t))}|\psi|^{2}\right)=0$ and $\frac{d}{d t}\left(\int_{\Omega \backslash B_{r}(x(t))} \psi\right)=0$ (Note that it will later be shown that $\frac{d}{d t}\|\nabla \psi\| \leq 0$, thus $\psi(t, \cdot) \in$ 
$H^{1}(\Omega)$ for all $t \geq 0$ if $\psi(0, \cdot) \in \Psi$ ). From (8), we have (with $\Omega(t)=\Omega \backslash B_{r}(x(t))$ ):

$$
\begin{aligned}
\frac{d}{d t}\left(\int_{\Omega(t)}|\psi|^{2}\right)= & 2 \int_{\Omega(t)} \psi \partial_{t} \psi+\mathbf{v} \cdot \int_{\partial B_{r}(x(t))}|\psi|^{2} \mathbf{n} \\
= & 2 \int_{\Omega(t)} \psi \Delta \psi+2 J(\psi) \int_{\Omega(t)}|\psi|^{2}+2 a(t) \times \\
& \int_{\Omega(t)}|\psi|^{2}+2 b(t) \int_{\Omega(t)} \psi+\mathbf{v} \cdot \int_{\partial B_{r}(x(t))}|\psi|^{2} \mathbf{n} \\
= & -2 \int_{\Omega(t)}|\nabla \psi|^{2}+2 J(\psi)+2 a(t) \\
& +\mathbf{v} \cdot \int_{\partial B_{r}(x(t))}|\psi|^{2} \mathbf{n} \\
= & 0,
\end{aligned}
$$

because $J(\psi)=\int_{\Omega(t)}|\nabla \psi|^{2}, \int_{\Omega(t)}|\psi|^{2}=1$ and $\int_{\Omega(t)} \psi=0($ since $\psi(t, \cdot) \in \Psi(x(t))$.) We also have:

$$
\begin{aligned}
\frac{d}{d t}\left(\int_{\Omega(t)} \psi\right)= & \int_{\Omega(t)} \partial_{t} \psi+\mathbf{v} \cdot \int_{\partial B_{r}(x(t))} \psi \mathbf{n} \\
= & \int_{\Omega(t)} \Delta \psi+J(\psi) \int_{\Omega(t)} \psi \\
& +a(t) \int_{\Omega(t)} \psi+b(|\Omega|-c) \\
& +\mathbf{v} \cdot \int_{\partial B_{r}(x(t))} \psi \mathbf{n} \\
= & 0 .
\end{aligned}
$$

Since we also have that $\psi(0, \cdot) \in \Psi(x(0))$, we conclude that the set $\Psi$ is invariant with respect to the dynamics (8).

Let $\{x(t), \psi(t, y)\}_{t \in \mathbb{R}_{\geq 0}}$ (with $y \in \Omega \backslash B_{r}(x(t))$ ), be a one-parameter family of functions that is a solution to the dynamics (8), and let $\psi(t, \cdot) \in \Psi(x(t))$ for all $t \in \mathbb{R}_{\geq 0}$ (this assumption is justified by the invariance of $\Psi)$. We have $J(\psi)=\int_{\Omega(t)}|\nabla \psi|^{2}=\frac{\int_{\Omega(t)}|\nabla \psi|^{2}}{\int_{\Omega(t)}|\psi|^{2}} \geq 0$ for $\psi(t, \cdot) \in$ $\Psi(x(t))$ (since $\left.\int_{\Omega(t)}|\psi|^{2}=1\right)$. Now:

$$
\begin{aligned}
\frac{d}{d t} J= & 2 \int_{\Omega(t)} \nabla \psi \cdot \nabla \partial_{t} \psi+\mathbf{v} \cdot \int_{\partial B_{r}(x(t))}|\nabla \psi|^{2} \mathbf{n} \\
& -2 J(\psi) \int_{\Omega(t)} \psi \partial_{t} \psi-J(\psi) \mathbf{v} \cdot \int_{\partial B_{r}(x(t))}|\psi|^{2} \mathbf{n} \\
= & -2 \int_{\Omega(t)}|\Delta \psi+J(\psi) \psi|^{2}-\mathbf{v} \cdot \mathbf{v}_{\text {int }} \leq 0,
\end{aligned}
$$

where we have used (8) to obtain the second equality. By the Rellich-Kondrachov Compactness Theorem [17, we see that the orbit $\psi$ is precompact in $L^{2}(\Omega)$. Thus, by the invariance 
principle [20], the solutions $\{x(t), \psi(t, y)\}_{t \in \mathbb{R}_{>0}}$ (with $y \in \Omega \backslash B_{r}(x(t))$ ), converge to $x^{*}, \psi^{*}$ (the convergence $\psi(t, \cdot) \rightarrow \psi^{*}$, is in the sense of $L^{2}$ ) such that $\mathbf{v}=0$ and $\Delta \psi^{*}+J\left(\psi^{*}\right) \psi^{*}=0$. We already have that the only asymptotically stable case is when $\psi^{*}=\psi_{2}^{*}$ (the second eigenfunction corresponding to $\left.\Omega \backslash B_{r}\left(x^{*}\right)\right)$, which implies that $J\left(\psi^{*}\right)=J\left(\psi_{2}^{*}\right)=\mu_{2}^{*}$. And $\mathbf{v}=0$ implies that $\int_{\partial B_{r}\left(x^{*}\right)}\left|\nabla \psi_{2}^{*}\right|^{2} \mathbf{n}=\mu_{2}^{*} \int_{\partial B_{r}\left(x^{*}\right)}\left|\psi_{2}^{*}\right|^{2} \mathbf{n}$, the critical point of the functional $\mu_{2}$ from (5).

Consider perturbations $\delta x$ and $\delta \psi$, about an equilibrium $\left(x^{*}, \psi_{2}^{*}\right)$ such that $x^{*}+\delta x \in \tilde{\Omega}$ and $\tilde{\psi}_{2}=\psi_{2}^{*}+\delta \psi \in \Psi\left(x^{*}+\delta x\right)$ is the second eigenfunction of the domain $\Omega \backslash B_{r}\left(x^{*}+\delta x\right)$. In other words, we consider perturbations purely in $x$ to investigate the local asymptotic stability of the critical points of $\mu_{2}(x)$. The dynamics in $x$ in this case, referring to (8), are given by:

$$
\frac{d}{d t}\left(x^{*}+\delta x\right)=-\int_{\partial B_{r}\left(x^{*}+\delta x\right)}\left(\left|\nabla \tilde{\psi}_{2}\right|^{2}-\tilde{\mu}_{2}\left|\tilde{\psi}_{2}\right|^{2}\right) \mathbf{n} .
$$

This can be reduced to:

$$
\frac{d}{d t}(\delta x)=-\left.\frac{\partial}{\partial x}\right|_{x=x^{*}}\left(\int_{\partial B_{r}(x)}\left(\left|\nabla \tilde{\psi}_{2}\right|^{2}-\tilde{\mu}_{2}|\tilde{\psi}|^{2}\right) \mathbf{n}\right) \delta x .
$$

From Equation (13), we recognize that $\int_{\partial B_{r}(x)}\left(\left|\nabla \tilde{\psi}_{2}\right|^{2}-\tilde{\mu}_{2}|\tilde{\psi}|^{2}\right) \mathbf{n}=\frac{\partial \mu_{2}}{\partial x}$. Therefore, the linearized dynamics reduces to:

$$
\frac{d}{d t}(\delta x)=-\left.\frac{\partial^{2} \mu_{2}}{\partial x^{2}}\right|_{x=x^{*}} \delta x
$$

where $\left.\frac{\partial^{2} \mu_{2}}{\partial x^{2}}\right|_{x=x^{*}}$ is the Hessian of $\mu_{2}$ at $x=x^{*}$. Therefore, we have that the linearized dynamics is asymptotically stable if and only if the Hessian of $\mu_{2}$ is positive definite, in other words, if and only if $x^{*}$ is a strict local minimum of $\mu_{2}$. Therefore, the necessary condition for the local asymptotic stability of the primal-dual dynamics at a critical point of $\mu_{2}$ is that it is a strict local minimum.

Proof. (Proof of Theorem 2). Let $r: \mathbb{R} \rightarrow \mathbb{R}_{\geq 0}$ with $r(0)=0$ be a smooth non-negative function. Let $\Omega(t)=\Omega \backslash B_{r(t)}(x)$ for some $x \in \Omega \subset \mathbb{R}^{N}$, be a one parameter family of spatial domains such that $\Omega(0)=\Omega$. Let $\mu_{2}(t)$ be the second eigenvalue of the domain $\Omega(t)$ and $\psi_{2}(t, \cdot)$ the corresponding normalized eigenfunction (we assume that the family of spatial domains $\Omega(t)$ have simple eigenvalues). Thus, we have $\mu_{2}(t)=\int_{x \in \Omega(t)}\left|\nabla \psi_{2}(t, x)\right|^{2}$. From [21], we have that $\mu_{2}(t)$ and $\psi_{2}$ are real-analytic locally at $t=0$. Thus, for small $\tau>0$, we have:

$$
\begin{aligned}
\mu_{2}(\tau) & =\mu_{2}(0)+\left.\frac{d}{d t} \mu_{2}\right|_{t=0} \tau+\ldots \\
\psi_{2}(\tau, x) & =\psi_{2}(0, x)+\left.\partial_{t} \psi_{2}(t, x)\right|_{t=0} \tau+\ldots
\end{aligned}
$$

We note that $\mu_{2}(0)$ and $\psi_{2}(0, \cdot)$ are the second eigenpair corresponding to $\Omega$. At a given $t>0$, let the deformation of the domain be characterized by $\mathbf{v}=-\epsilon \mathbf{n}$, the velocity of points 
on the boundary of the hole, $B_{r(t)}(x)$, where $\mathbf{n}$ is the normal to the domain $\Omega(t)$ on the boundary of $B_{r(t)}(x)$, and $\epsilon>0$ is a small constant. We have:

$$
\begin{aligned}
\frac{d}{d t} \mu_{2}= & \frac{d}{d t} \int_{x \in \Omega(t)}\left|\nabla \psi_{2}(t, x)\right|^{2} \\
= & 2 \int_{\Omega(t)} \nabla \psi_{2} \nabla \partial_{t} \psi_{2}+\int_{\partial B_{r(t)}(x)}\left|\nabla \psi_{2}\right|^{2} \mathbf{v} \cdot \mathbf{n} \\
= & -2 \int_{\Omega(t)} \Delta \psi_{2} \partial_{t} \psi_{2}+\int_{\partial B_{r(t)}(x)}\left|\nabla \psi_{2}\right|^{2} \mathbf{v} \cdot \mathbf{n} \\
= & 2 \mu_{2}(t) \int_{\Omega(t)} \psi_{2} \partial_{t} \psi_{2}+\int_{\partial B_{r(t)}(x)}\left|\nabla \psi_{2}\right|^{2} \mathbf{v} \cdot \mathbf{n} \\
= & \mu_{2}(t)\left(\frac{d}{d t} \int_{\Omega(t)}\left|\psi_{2}\right|^{2}-\int_{\partial B_{r(t)}(x)}\left|\psi_{2}\right|^{2} \mathbf{v} \cdot \mathbf{n}\right) \\
& +\int_{\partial B_{r(t)}(x)}\left|\nabla \psi_{2}\right|^{2} \mathbf{v} \cdot \mathbf{n} \\
= & \mu_{2}(t) \epsilon \int_{\partial B_{r(t)}(x)}\left|\psi_{2}\right|^{2}-\epsilon \int_{\partial B_{r(t)}(x)}\left|\nabla \psi_{2}\right|^{2}
\end{aligned}
$$

since $\int_{\Omega(t)}\left|\psi_{2}\right|^{2}=1$, for all $t$. For small $\tau>0$, we then substitute from (15) in the above equation, to obtain:

$$
\begin{aligned}
\frac{d}{d t} \mu_{2}= & \epsilon\left(\mu_{2}(0)+\left.\frac{d}{d t} \mu_{2}\right|_{t=0} \tau+\ldots\right) \times \\
& \int_{y \in \partial B_{r(\tau)}(x)}\left|\psi_{2}(0, y)+\partial_{t} \psi_{2}(t, y)\right|_{t=0} \tau+\left.\ldots\right|^{2} \\
& -\epsilon \int_{y \in \partial B_{r(\tau)}(x)}\left|\nabla\left(\psi_{2}(0, y)+\left.\partial_{t} \psi_{2}(t, y)\right|_{t=0} \tau+\ldots\right)\right|^{2} \\
= & \mu_{2}(0) \epsilon \int_{y \in \partial B_{r(\tau)}(x)}\left|\psi_{2}(0, y)\right|^{2}-\epsilon \int_{y \in \partial B_{r(\tau)}(x)}\left|\nabla \psi_{2}(0, y)\right|^{2} \\
& +\mathcal{O}(\tau) \\
= & \mu_{2}(0) S_{N-1} r(\tau)^{N-1} \epsilon\left|\psi_{2}(0, x)\right|^{2} \\
& -S_{N-1} r(\tau)^{N-1} \epsilon\left|\nabla \psi_{2}(0, x)\right|^{2}+\mathcal{O}\left(r(\tau)^{N-1} \tau\right)
\end{aligned}
$$

where $S_{N}$ is the surface area of th unit $N$-sphere. Now, given that $\mathbf{v}=-\epsilon \mathbf{n}$, we have $r(\tau)=\epsilon \tau$, and therefore:

$$
\begin{aligned}
\frac{d}{d t} \mu_{2}= & \mu_{2}(0) S_{N-1} \epsilon^{N} \tau^{N-1}\left|\psi_{2}(0, x)\right|^{2} \\
& -S_{N-1} \epsilon^{N} \tau^{N-1}\left|\nabla \psi_{2}(0, x)\right|^{2}+\mathcal{O}\left(\tau^{N}\right)
\end{aligned}
$$

Substituting for $\frac{d}{d t} \mu_{2}$ from the above equation into $\mu_{2}(\tau)=\mu_{2}(0)+\left.\frac{d}{d t} \mu_{2}\right|_{\bar{\tau}} \tau$ (where $\bar{\tau} \in[0, \tau]$ ), 
we get:

$$
\begin{aligned}
\mu_{2}(\tau)= & \mu_{2}(0)+S_{N-1} \epsilon^{N} \bar{\tau}^{N-1} \tau\left(\mu_{2}(0)\left|\psi_{2}(0, x)\right|^{2}-\left|\nabla \psi_{2}(0, x)\right|^{2}\right) \\
& +\mathcal{O}\left(\bar{\tau}^{N} \tau\right) \\
\leq & \mu_{2}(0)+S_{N-1} \epsilon^{N} \tau^{N}\left(\mu_{2}(0)\left|\psi_{2}(0, x)\right|^{2}-\left|\nabla \psi_{2}(0, x)\right|^{2}\right) \\
& +\mathcal{O}\left(\tau^{N+1}\right) \\
\approx & \mu_{2}(0)+c(\tau)\left(\mu_{2}(0)\left|\psi_{2}(0, x)\right|^{2}-\left|\nabla \psi_{2}(0, x)\right|^{2}\right)
\end{aligned}
$$

where we have ignored the $\mathcal{O}\left(\tau^{N+1}\right)$ term in the final expression. We also have $r(\tau)=\epsilon \tau$, and therefore the above can also be written as $\mu_{2}(r) \approx \mu_{2}(0)+c(r)\left(\mu_{2}(0)\left|\psi_{2}(0, x)\right|^{2}-\left|\nabla \psi_{2}(0, x)\right|^{2}\right)$ as a function of the radius of the hole. We also note that the function $\left(\mu_{2}(0)\left|\psi_{2}(0, x)\right|^{2}-\left|\nabla \psi_{2}(0, x)\right|^{2}\right)=$ $\lim _{r \rightarrow 0} \frac{1}{\left|\partial B_{r}(x)\right|} \frac{\partial}{\partial r} \mu_{2}\left(\Omega \backslash B_{r}(x)\right)$.

We now show that the local minima of $f(x)=\mu_{2}^{\Omega}\left|\psi_{2}^{\Omega}\right|^{2}-\left|\nabla \psi_{2}^{\Omega}\right|^{2}$ occur along the nodal set of $\psi_{2}^{\Omega}$, that is, in the set $\left\{x \in \Omega \mid \psi_{2}^{\Omega}(x)=0\right\}$, in the region where the family of level sets of $\psi_{2}^{\Omega}$ is locally flat. Let $\left\{\mathbf{r}, \mathbf{t}_{1}, \ldots, \mathbf{t}_{N-1}\right\}$ be an orthonormal basis at $x \in \Omega$, where $\mathbf{r}$ is the unit normal to the level set of $\psi_{2}^{\Omega}$ at $x$ and $\left\{\mathbf{t}_{1}, \ldots, \mathbf{t}_{N-1}\right\}$ the unit tangents. We can express the gradient operator in this coordinate system as $\nabla=\mathbf{r} \frac{\partial}{\partial r}+\sum_{i=1}^{N-1} \mathbf{t}_{i} \frac{\partial}{\partial t_{i}}$. We now have $\nabla \psi_{2}^{\Omega}=\frac{\partial \psi_{2}^{\Omega}}{\partial r} \mathbf{r}$ (since the derivative of $\psi_{2}^{\Omega}$ vanishes along the tangent space of its level set). Moreover, the eigenvalue equation $\Delta \psi_{2}^{\Omega}+\mu_{2}^{\Omega} \psi_{2}^{\Omega}=0$ expressed in this coordinate system is given by $\frac{\partial^{2} \psi_{2}^{\Omega}}{\partial r^{2}}+(N-1) H \frac{\partial \psi}{\partial r}+\mu_{2}^{\Omega} \psi_{2}^{\Omega}=0$, where $H(x)$ is the mean curvature at $x \in \Omega$ of the level set of $\psi$. Following some computation, we get that the gradient of $f$ is given by $\nabla f=4 \mu_{2}^{\Omega} \psi_{2}^{\Omega} \frac{\partial \psi_{2}^{\Omega}}{\partial r} \mathbf{r}+2(N-1) H\left|\frac{\partial \psi_{2}^{\Omega}}{\partial r}\right|^{2} \mathbf{r}$. Moreover, in computing the entries of the Hessian of $f$ in this coordinate frame, we first have:

$$
\begin{aligned}
\frac{\partial^{2} f}{\partial r^{2}}=4 \mu_{2}^{\Omega}\left(\left|\frac{\partial \psi_{2}^{\Omega}}{\partial r}\right|^{2}-\mu_{2}^{\Omega}\left|\psi_{2}^{\Omega}\right|^{2}\right) & -8(N-1) H \mu_{2}^{\Omega} \psi_{2}^{\Omega} \frac{\partial \psi_{2}^{\Omega}}{\partial r} \\
& +\left(2(N-1) \frac{\partial H}{\partial r}-4(N-1)^{2} H^{2}\right)\left|\frac{\partial \psi_{2}^{\Omega}}{\partial r}\right|^{2}
\end{aligned}
$$

Clearly, for any point $x^{*}$ where the family of level sets of $\psi_{2}^{\Omega}$ is locally flat (which in particular implies $\left.H\left(x^{*}\right)=0\right)$, we have that $x^{*}$ is a critical point if and only if $\psi_{2}^{\Omega}\left(x^{*}\right)=0$ or $\nabla \psi_{2}^{\Omega}\left(x^{*}\right)=$ 0 . Furthermore, we have $\frac{\partial^{2} f}{\partial r^{2}}\left(x^{*}\right)=4 \mu_{2}^{\Omega}\left(\left|\frac{\partial \psi_{2}^{\Omega}}{\partial r}\left(x^{*}\right)\right|^{2}-\mu_{2}^{\Omega}\left|\psi_{2}^{\Omega}\left(x^{*}\right)\right|^{2}\right)$. Also, under local flatness of the family of level sets, the off-diagonal entries $\frac{\partial^{2} f}{\partial r \partial t_{i}}$ and $\frac{\partial^{2} f}{\partial t_{i} \partial t_{j}}$ vanish for all $i \in\{1, \ldots, N-1\}$, and so do the rest of the diagonal entries of the Hessian, i.e. $\frac{\partial^{2} f}{\partial t_{i}^{2}}\left(x^{*}\right)=0$ for $i \in\{1, \ldots, N-1\}$. It thereby follows that the Hessian is positive semidefinite when $\psi_{2}^{\Omega}\left(x^{*}\right)=0$ and negative semidefinite when $\nabla \psi_{2}^{\Omega}\left(x^{*}\right)=0$. Therefore, under local flatness of the family of level sets of $\psi_{2}^{\Omega}$, the nodal points of $\psi_{2}^{\Omega}$ correspond to the local minima of $f$.

\section{References}

[1] A. Jamakovic and P. V. Mieghem, "On the robustness of complex networks by using the algebraic connectivity," in International conference on research in networking. Springer, 
2008, pp. $183-194$.

[2] M. Fiedler, "Algebraic connectivity of graphs," Czechoslovak Mathematical Journal, vol. 23, no. 98, pp. 298-305, 1973.

[3] M. Ventresca and D. Aleman, "Efficiently identifying critical nodes in large complex networks," Computational Social Networks, vol. 2:6, no. 1, 2015. [Online]. Available: https://doi.org/10.1186/s40649-015-0010-y

[4] A. Arulselvan, C. Commander, L. Elefteriadou, and P. Pardalos, "Detecting critical nodes in sparse graphs," Computers and Operations Research, vol. 36, no. 7, pp. 2193$2200,2009$.

[5] W. Abbas, A. Laszka, Y. Vorobeychik, and X. Koutsoukos, "Improving network connectivity using trusted nodes and edges," in American Control Conference, Seattle, USA, 2017, pp. 328-333.

[6] M. Sheng, J. Li, and Y. Shi, "Critical nodes detection in mobile ad hoc network," in Int. Conf. on Advanced Information Networking and Applications, vol. 2. IEEE, 2006, pp. $336-340$.

[7] K. P. Eswaran and R. E. Tarjan, "Augmentation problems," SIAM Journal on Computing, vol. 5, no. 4, pp. 653-665, 1976.

[8] M. Dziubiński and S. Goyal, "Network design and defence," Games and Economic Behavior, vol. 79, pp. 30-43, 2013.

[9] L. Lovász, Large networks and graph limits, ser. Coloquium Publications. American Mathematical Society, 2012, vol. 60.

[10] M. Belkin and P. Niyogi, "Towards a theoretical foundation for Laplacian-based manifold methods," in Int. Conf. on Computational Learning Theory. Springer, 2005, pp. 486500 .

[11] _ - "Convergence of Laplacian eigenmaps," Advances in Neural Information Processing Systems, vol. 19, pp. 129-136, 2007.

[12] A. Henrot, Extremum problems for eigenvalues of elliptic operators. Springer Science \& Business Media, 2006.

[13] T. Kolokolnikov, M. S. Titcombe, and M. J. Ward, "Optimizing the fundamental Neumann eigenvalue for the Laplacian in a domain with small traps," European Journal of Applied Mathematics, vol. 16, no. 2, pp. 161-200, 2005.

[14] V. Krishnan and S. Martínez, "Identification of critical nodes for consensus in large-scale spatial networks," in IFAC World Congress, Toulouse, France, July 2017, pp. 1472114726.

[15] P. Goyal and E. Ferrara, "Graph embedding techniques, applications, and performance: A survey," arXiv preprint arXiv:1705.02801, 2017. 
[16] B. Shaw and T. Jebara, "Structure preserving embedding," in Proceedings of the 26th Annual International Conference on Machine Learning. ACM, 2009, pp. 937-944.

[17] L. C. Evans, Partial differential equations, ser. Graduate studies in mathematics. Providence, RI: American Mathematical Society, 1998.

[18] R. Murray, B. Swenson, and S. Kar, "Revisiting normalized gradient descent: Evasion of saddle points," arXiv preprint arXiv:1711.05224, 2017.

[19] R. Atar and K. Burdzy, "On nodal lines of Neumann eigenfunctions," Electronic Communications in Probability, vol. 7, pp. 129-139, 2002.

[20] D. Henry, Geometric theory of semilinear parabolic equations. Springer, 1981.

[21] M. L. de Cristoforis, "Simple Neumann eigenvalues for the Laplace operator in a domain with a small hole," Revista Matematica Complutense, vol. 25, no. 2, pp. 369-412, 2012. 